\title{
Unusual Primary Ocular Manifestation of Sarcoidosis
}

\author{
Svenja Deuchler ${ }^{a}$ Clemens Wagner $^{b} \quad$ Markus Schill $^{b} \quad$ Benjamin Schöne ${ }^{b}$ \\ Daniel Scherer $^{\mathrm{b}}$ Pankaj Singh ${ }^{\mathrm{a}}$ Thomas Kohnen $^{\mathrm{c}}$ Frank H.J. Koch ${ }^{\mathrm{a}}$ \\ aRetina and Vitreous Unit, University Eye Clinic, Frankfurt, Germany; ${ }^{b}$ VRmagic, \\ Mannheim, Germany; ' University Eye Clinic, Frankfurt, Germany
}

\section{Keywords}

Sarcoidosis · Optic disc edema $\cdot$ Eyesi $\cdot$ Simulation $\cdot$ Education

\section{Abstract}

A 23-year-old male patient presented with very mild visual disturbances, but a distinct prominence of the optic discs, more pronounced in the right than in the left eye. The ophthalmic symptoms initially seemed trivial, but a large-scale interdisciplinary workup later identified them as the presenting symptoms of sarcoidosis affecting lung and eyes. A standard steroid monotherapy successfully caused regression of the ophthalmic findings.

(C) 2019 The Author(s)

Published by S. Karger AG, Basel

\section{Introduction}

Sarcoidosis is a systemic, multi-organ disease that exists all over the world, but is more common in colder climates. Typically, it occurs in younger ages, heritability as well as an association with infectious agents is controversially discussed. Sarcoidosis can have a variety of primary manifestations with a frequent involvement of lungs and lymph nodes. It can remain undetected for a long time, but its progression can be fatal.

Regarding ocular manifestations, granulomatous anterior uveitis (acute or chronic) is most common. Posterior segment lesions are present in no more than 20\%. During the International Workshop On Ocular Sarcoidosis (IWOS) in 2009, an international group of uveitis 


\section{Case Reports in Ophthalmology}

specialists determined seven signs for the diagnosis of intraocular sarcoidosis [1]: Mutton-fat or small granulomatous keratic precipitates and/or iris nodules (Koeppe/Busacca); trabecular meshwork nodules and/or tent-shaped peripheral anterior synechiae; vitreous opacities displaying snowballs/"strings of pearls"; multiple chorioretinal peripheral lesions (active and/or atrophic); nodular and/or segmental periphlebitis with or without candlewax drippings and/or retinal macroaneurysm in an inflamed eye; optic disc nodule(s)/granuloma(s) and/or solitary choroidal nodule as well as bilaterality. There is no standardized treatment pattern [2], therefore the analysis of individual cases plays an important role.

We describe the case of a 23-year-old male patient who presented with minimal visual disturbances but bilateral optic disc edema, pronounced in the right eye. Only after a thorough interdisciplinary workup, we could identify this condition as an unusual primary manifestation of a sarcoidosis with ocular and lung involvement.

Based on this case, we designed an extension to a simulator-based curriculum, relevant for the education of medical students and residents (see the Discussion section).

\section{Case Report}

A 23-year-old male patient who had previously been healthy presented at the university eye clinic complaining about slightly blurred vision and "some spots" in his right visual field. Best-corrected visual acuity (BCVA) was 0.7 in the right and 1.0 in the left eye. Ophthalmic examination showed a mild prominence of the disc in the left eye and a distinct prominence with cotton wool spots and tortuous retinal vessels in the right eye (Fig. 1a, b). Additionally cotton wool-like spots/perivascular sheathing (Fig. 1c), inflammatory cells in the anterior chamber as well as in the vitreous body and cell deposits at the corneal endothelium were found - unmistakably findings of intraocular inflammation (consistent with uveitis and vasculitis in the vitreous and at the disc). Case workup (cooperation of ophthalmology with neurology and internal medicine) concentrated on different pathologies that were associated with a prominent disc and on diagnostic ways to achieve a differential diagnosis (papillitis, papilledema, encephalitis, sinus vein thrombosis, infarcts, intracranial hemorrhages, tumors, pseudotumor cerebri, hypertensive retinopathy [stage IV], Vogt-Koyanagi-Harada syndrome, and multiple sclerosis). A contrast cMRT was performed and encephalitis, sinus vein thrombosis, recent infarcts, and intracranial hemorrhages were excluded.

Blood pressure was regular. The cerebrospinal fluid (CSF) opening pressure values were normal $(21 \mathrm{~cm} \mathrm{H2O})$ as well as the liquor spaces in CCT and cMRI, thus a pseudotumor cerebri could be excluded. Initial blood findings were unspectacular except for strongly increased D-dimers of $895 \mathrm{ng} / \mathrm{mL}$ (norm <500) and calcium in blood $2.62 \mathrm{mmol} / \mathrm{L}$ (norm 2.09-2.54). The CSF analysis revealed a leukocytosis of $48 / \mu \mathrm{L}$ (norm $0-4$ ), a slightly increased total protein of $571 \mathrm{mg} / \mathrm{L}$ (norm <450) and slightly increased immunoglobulins: immunoglobulin G $45.3 \mathrm{mg} / \mathrm{L}$ (norm 10-40) and immunoglobulin A $7.1 \mathrm{mg} / \mathrm{L}$ (norm 0.5-6). Lactate with 2.24 $\mathrm{mmol} / \mathrm{L}$ (norm 1.1-2.4) and glucose with $43.3 \mathrm{mg} / \mathrm{dL}$ (norm 40-70) were normal. Inflammatory liquor changes of viral genesis were suspected, therefore, we decided to determine neurotropic pathogens (in serum and CSF) and initiated extended diagnostic procedures to find a reason for the vasculitis. While waiting for further blood serology results, a virostatic agent (acyclovir $500 \mathrm{mg}$ i.v. three times a day) and an antibiotic drug (cefotaxime $2.0 \mathrm{~g}$ three times a day) were given. Additional systemic steroids were considered based on serology values.

Three days later the retinal examination with OCT detected a detachment of the neurosensory retina in the macula suspicious of being connected to an optic disc edema (Fig. 1d). In 
addition, the ensheathing of veins with perivascular infiltrates in the fundus appeared to be progressive. In the patient's left eye, the disc was still less prominent than in the right eye and the macula was dry. Fluorescein angiography could not be performed because of the patient's circulatory instability. As the cause of the disc edema was still unknown, the workup was extended: a chest X-ray (Fig. 2a, b) was performed and additional blood testing was initiated.

The X-rays (Fig. 2a, b) showed bihilar shading with donut sign, compatible with a lymphadenopathy, plus bilateral, basally pronounced streaky pulmonary lung patterns. Serology findings were negative for neurotropic pathogens, borreliosis, HSV, CMV, EBV, VZV, HIV, tuberculosis, rheumatic factor, ANA, and ANCA. Coagulation factors, antiphospholipids and liver enzymes were normal and there were no signs of thrombophilia.

Blood results that matched with a sarcoidosis included an increase of $\mathrm{IL}_{2} \mathrm{R}: 1,681 \mathrm{U} / \mathrm{mL}$ (norm 158-623), increased erythrocyte sedimentation rate of $20 \mathrm{~mm} / \mathrm{h}$ (norm $<15$ ), and increased $\mathrm{IL}_{2} \mathrm{R}$ in the CSF with $99.2 \mathrm{U} / \mathrm{mL}$ (norm $<8$ ). With $42.0 \mathrm{U} / \mathrm{L}$, the ACE level was in the high-end normal range (8-52). In an extracranial and transcranial Doppler sonography, there were no signs for a vasculitis or clinically relevant vascular stenosis. Spirometry showed a slightly restricted ventilatory disorder. This was a key situation: the additional blood tests and the X-ray findings were compatible with a sarcoidosis type II. Chest CT showed mediastinal and bihilar enlarged lymph nodes confirming the diagnosis of sarcoidosis (Fig. 2c-f). Thoracic endosonography (endobronchial) with needle aspiration excluded lymphoma, tuberculosis, and malignant processes and gave histopathological confirmation of the diagnosis of sarcoidosis. The bronchoalveolar lavage fluid (BALF) showed the characteristic elevation in the CD4/CD8 T-cell ratio. A therapy with prednisolone $250 \mathrm{mg}$ i.v. was started for 3 days, then $100 \mathrm{mg}$ prednisolone 1-0-0 was administered orally.

After 2 weeks of treatment, a decrease in the optic disc edemas on both sides and an almost reconstituted macula in the right eye was found. The retinal deposits in the periphery suspicious of vasculitis were scarce on both sides. The subjective symptoms also improved, and the BCVA was 0.8 in the right eye and continued to be stable with 1.0 in the left eye.

With the help of optic coherence tomography (OCT-retina) and fluorescein angiography, retinal granulomas could be detected (Fig. 3a, b).

Three weeks later, the low amount of residual disc swelling and perivascular infiltrations allowed reduction of the steroids every 10 days down to $10 \mathrm{mg}$ per day. At the 3-month followup, there were no signs of a vasculitis anymore and the $\mathrm{IL}_{2} \mathrm{R}$ control parameter was down to $50 \%$ of the initial value. Since there was no recurrence, the steroids could be reduced to a maintenance dose.

After 2 months of steroid treatment on a low maintenance dose, the patient presented with no subjective symptoms and full BCVA, mild residual prominence of the disc (Fig. 3c, d), reduced sheathing of the vessels (Fig. $3 \mathrm{e}$ ) and a fully attached macula (Fig. 3f). The $\mathrm{IL}_{2} \mathrm{R}$ profile (norm 158-623 U/mL) was evaluated over time: 1,681 U/mL (initial presentation, - 765 $759-615-446-498$ under steroid maintenance dose) and further monitored to decide about the need to continue steroid therapy.

One year after the first presentation, the patient had full vision (cc 1.25) with no visual disturbances. There was no relapse of the initial ophthalmic findings, but still increased tortuosity of the retinal vessels; f/u X-ray was performed and still showed enlarged lymph nodes. $\mathrm{IL}_{2} \mathrm{R}$ was slightly increased to $579 \mathrm{U} / \mathrm{mL}$ but remained in the normal range. Both parameters together with a constantly increased calcium blood level (ongoing production of 1.25-dihydroxy-cholecalciferol by macrophages in granulomas) and intermittent coughing implied that the steroid therapy should be continued. Due to the long intake of steroids, the patient's bone density was reduced, therefore the steroid dose was lowered to $2.5 \mathrm{~mL} /$ day and the 
monitoring interval tightened. Because of the improvement of symptoms, we applied a waitand-see behavior regarding a steroid sparing immunosuppressive therapy at that time.

Lately, 17 months after initial presentation, the patient was stable without new ocular symptoms and signs under a steroid dose of $2.5 \mathrm{~mL} /$ day. X-rays showed no progression of disease and the $\mathrm{IL}_{2} \mathrm{R}$ level decreased again, so all disciplines involved into the treatment agreed to start an attempt to withdraw the steroids completely with an ongoing tight monitoring interval.

In summary, it is important to retain a constant treatment period of at least 6 months to avoid a relapse. During this time, the steroid dose should be adapted to the clinical course of disease and should not fall short of the maintenance dose. In case of aggravation of the disease or ongoing steroid-related adverse events, a long-term steroid sparing therapy with, for example, methotrexate should be initiated.

\section{Discussion/Conclusion}

Optic disc edema is an unusual, but not unknown presentation of sarcoidosis [3]. Typically, the swelling of the optic disc is associated with an increase of ICP (and then called papilledema) with its various causes (e.g., space-occupying intracranial lesions or cerebral edema, disturbances of the CSF flow or intracranial hypertension) and associated pathologies (e.g., meningitis, hydrocephalus, brain tumors, pseudotumor cerebri [4]); a more exotic example [5] describes papilledema as an initial presentation of Castleman disease. Sarcoidosis and other inflammatory or infectious processes (e.g., syphilis [6], HIV-associated meningoradiculitis [7] may induce perineuritis and, as a consequence, an optic disc swelling without increased ICP $[8,9])$.

There are many causes leading to the development of an optic disc edema, as there are many clinical pictures leading to sarcoidosis. The disease can manifest itself in every organ [10], with a strong pulmonal preference [11]. Up to $80 \%$ of all sarcoidosis patients develop an ocular involvement during the course of the disease [12]. As mentioned above, the Intraocular Workshop on Ocular Sarcoidosis [1] lists seven typical ophthalmic findings. In its revised version [13] this guideline still does not include optic disc edema. As a primary manifestation of sarcoidosis this finding is quite uncommon, but should not be forgotten.

Our team is largely involved in the development of simulator-based teaching formats and technologies $[14,15]$. During the design of the curriculum for the ophthalmoscope simulator Eyesi indirect $[16,17]$, it became clear that a simulator-based curriculum can go beyond the simple presentation of visual findings and the associated pattern recognition. In the past, we developed a variety of complex cases where the simulated patients present themselves as in daily clinical life so that the trainee can learn to associate his/her observations with the complex manifestation of real-life pathologies.

However, all these cases are presented as a snapshot in time. For the case at hand, this is inadequate because the right diagnosis could only be found after several examination stages. Therefore, we are currently developing a new teaching format where patients repeatedly present themselves within an extended period of time. The sarcoidosis case can serve as a valuable example to teach that it might be necessary to amplify standard diagnosis and treatment patterns and to pursue less obvious, probably interdisciplinary pathways. 


\section{Statement of Ethics}

This case complies with the tenets of the Declaration of Helsinki. The patient has given written informed consent for publication of this case report.

\section{Disclosure Statement}

Clemens Wagner and Markus Schill are shareholders of and employed by VRmagic, a commercial company. Benjamin Schöne and Daniel Scherer are employed by VRmagic.

\section{Author Contributions}

Svenja Deuchler: coordinator of the patient's examinations, treatment procedures, and follow-ups. Clemens Wagner: coordinator of the technical development of the Eyesi simulator. Markus Schill: CEO of VRmagic, concept of stage-based case format. Benjamin Schöne: language editor for the text-based content in the Eyesi simulator. Daniel Scherer: graphical modeling for the Eyesi simulator. Pankaj Singh: interdisciplinary coordination. Thomas Kohnen: head of the ophthalmology department. Frank Koch: medical advisor of the retina and vitreous unit.

\section{References}

1 Herbort CP, Rao NA, Mochizuki M; members of Scientific Committee of First International Workshop on Ocular Sarcoidosis. International criteria for the diagnosis of ocular sarcoidosis: results of the first International Workshop On Ocular Sarcoidosis (IWOS). Ocul Immunol Inflamm. 2009 May-Jun;17(3):160-9.

2 Hebel R, Dubaniewicz-Wybieralska M, Dubaniewicz A. Overview of neurosarcoidosis: recent advances. J Neurol. 2015 Feb;262(2):258-67.

3 Bowling B. Kanski's Clinical Ophthalmology. A systematic approach. 8th ed. Amsterdam: Elsevier; 2016. pp. 420-4.

4 Bienfang DC. Papilledema. In: Ettinger AB, Weisbrot DM, editors. Neurologic Differential Diagnosis: A CaseBased Approach. Cambridge: Cambridge University Press; 2014. pp. 332-6.

5 Tian G, Jing Y, Jiang H, Wang J, Zhang X. Papilledema as the initial presentation of Castleman disease. J Neuroophthalmol. 2014 Jun;34(2):169-72.

6 Yri H, Wegener M, Jensen R. Syphilis mimicking idiopathic intracranial hypertension. BMJ Case Rep. 2011 Nov;2011 nov08 1:bcr0920114813.

7 Mandviwala MM, Amram AL, Rigi M. Papilledema. In: Smith SV, Lee AG, Brazis PW, editors. Clinical Pathways in Neuro-Ophthalmology: An Evidence-Based Approach. 3rd ed. New York: Thieme; 2018. pp. 89-110.

8 Katz JM, Bruno MK, Winterkorn JM, Nealon N. The pathogenesis and treatment of optic disc swelling in neurosarcoidosis: a unique therapeutic response to infliximab. Arch Neurol. 2003 Mar;60(3):426-30.

9 Koczman JJ, Rouleau J, Gaunt M, Kardon RH, Wall M, Lee AG. Neuro-ophthalmic sarcoidosis: the University of Iowa experience. Semin Ophthalmol. 2008 May-Jun;23(3):157-68.

10 Samuels MA, Gonzalez RG, Makadzange AT, Hedley-Whyte ET. Case 3-2017. A 62-Year-Old Man with Cardiac Sarcoidosis and New Diplopia and Weakness. N Engl J Med. 2017 Jan;376(4):368-79.

11 Criado E, Sánchez M, Ramírez J, Arguis P, de Caralt TM, Perea RJ, et al. Pulmonary sarcoidosis: typical and atypical manifestations at high-resolution CT with pathologic correlation. Radiographics. 2010 Oct;30(6):1567-86.

12 Pasadhika S, Rosenbaum JT. Ocular Sarcoidosis. Clin Chest Med. 2015 Dec;36(4):669-83.

13 Mochizuki M, Smith JR, Takase H, Kaburaki T, Rao NA. Revised International Criteria for the Diagnosis of Ocular Sarcoidosis. Invest Ophthalmol Vis Sci. 2018;59:4191.

14 Schuppe O, Wagner C, Koch F, Männer R. EYESi ophthalmoscope - a simulator for indirect ophthalmoscopic examinations. Stud Health Technol Inform. 2009;142:295-300. 


\section{Case Reports in Ophthalmology}

\begin{tabular}{l|l}
\hline Case Rep Ophthalmol 2019;10:205-212 \\
\hline DOI: 10.1159/000501302 & $\begin{array}{l}\text { @ 2019 The Author(s). Published by S. Karger AG, Basel } \\
\text { www.karger.com/cop }\end{array}$ \\
\hline
\end{tabular}

Deuchler et al.: Unusual Primary Ocular Manifestation of Sarcoidosis

15 Deuchler S, Wagner C, Singh P, Müller M, Al-Dwairi R, Benjilali R, et al. Clinical efficacy of simulated vitreoretinal surgery to prepare surgeons for the upcoming intervention in the operating room. PLoS One. 2016 Mar;11(3):e0150690.

16 Ting DS, Sim SS, Yau CW, Rosman M, Aw AT, Yeo IY. Ophthalmology simulation for undergraduate and postgraduate clinical education. Int J Ophthalmol. 2016 Jun;9(6):920-4.

17 Chou J, Kosowsky T, Payal AR, Gonzalez Gonzalez LA, Daly MK. Construct and face validity of the EYESI indirect opthalmoscope simulator. Retina. 2017 Oct;37(10):1967-76.
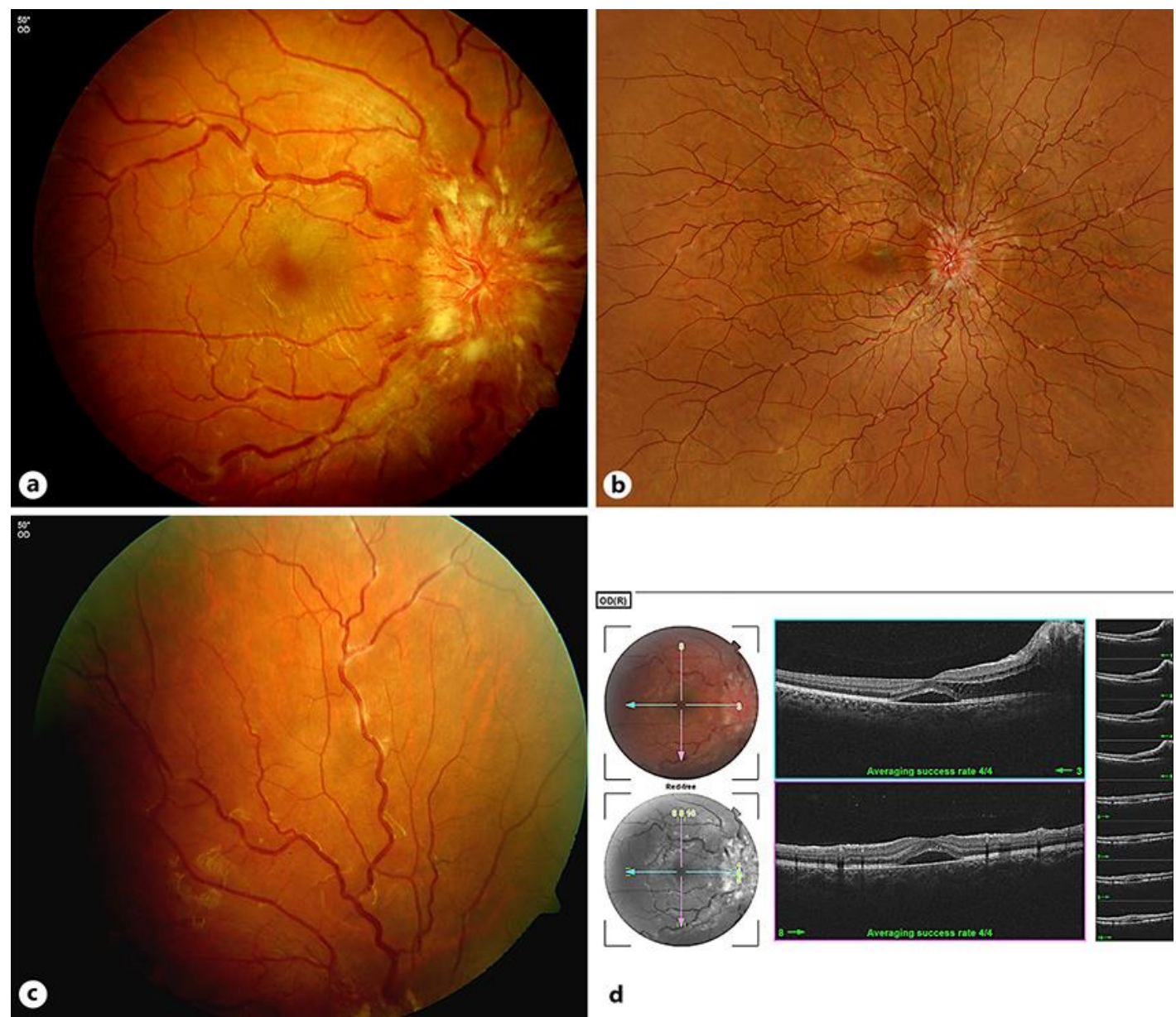

Fig. 1. Real and virtual examination results, initial stage. a, c As shown here for initial stage, real fundus images are stitched together and enhanced for the simulator in a manual process. $\mathbf{b}$ The resulting panoramic retina is then projected onto the three-dimensional model of the eye of the virtual patient. $\mathbf{d}$ The simulated ophthalmoscopic examination is complemented by additional diagnostic means, such as OCT, which are presented by the teaching software of the simulator. 


\section{Case Reports in Ophthalmology}

\begin{tabular}{l|l}
\hline Case Rep Ophthalmol 2019;10:205-212 \\
\hline DOI: 10.1159/000501302 & $\begin{array}{l}\text { ○ 2019 The Author(s). Published by S. Karger AG, Basel } \\
\text { www.karger.com/cop }\end{array}$ \\
\hline
\end{tabular}
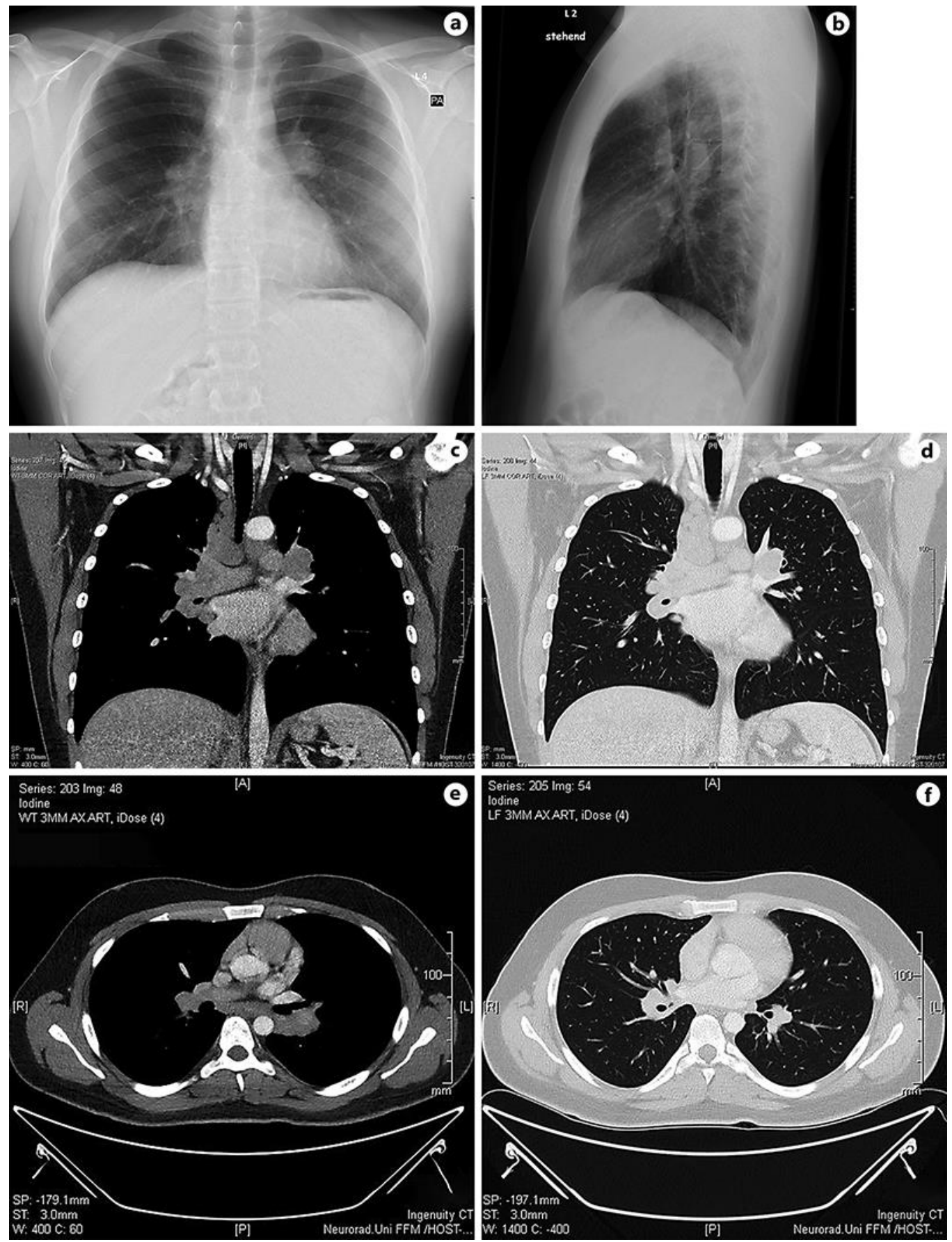

Fig. 2. Results of X-ray and CT imaging. X-rays show bihilar shading and bilateral streaky patterns. The chest CTs confirm mediastinal and bihilar enlarged lymph nodes. Image details: a PA X-ray; b lateral X-ray; c soft-tissue window CT with contrast agent (iodine), coronal cut; $\mathbf{d}$ lung window, coronal cut; e soft-tissue window, axial cut, and f lung window, axial cut. 


\section{Case Reports in Ophthalmology}
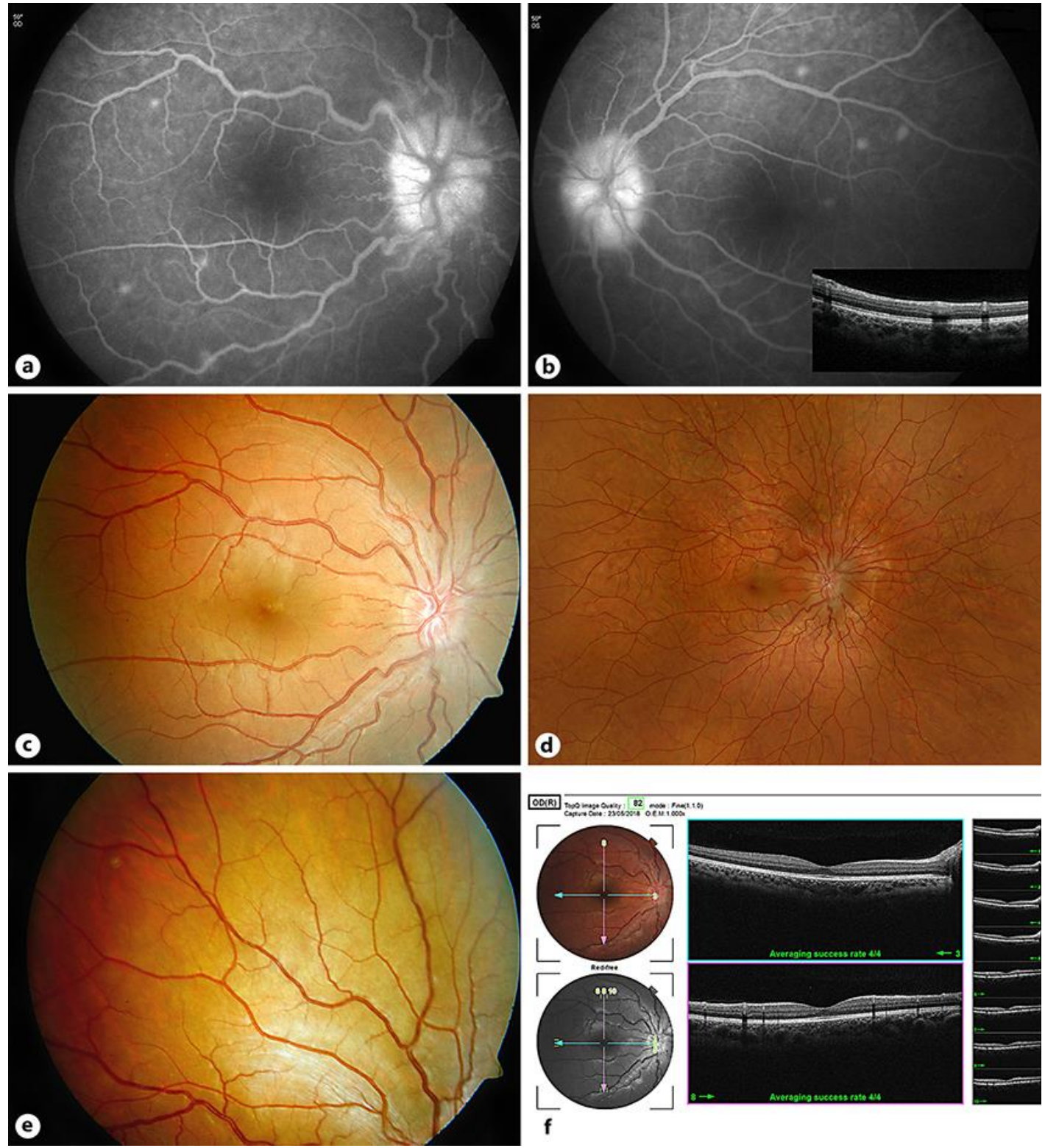

Fig. 3. Real and virtual examination results with: a onset of retinal granulomas $0 / \mathrm{U}$, fluorescein angiography; b fluorescein angiography/OCT; c regression of disc edema in reality; $\mathbf{d}$ regression of pathology in Eyesi Indirect; e regression of vascular tortuosity and sheathing in the upper temporal quadrant, and (f) complete regression of macula edema and subtotal reduction of optic disc swelling. 